\title{
TEM Study of Microstructure of 316L Stainless Steel with Different Specimen Preparation Techniques
}

\author{
Sz-Chian Liou ${ }^{1}$ and Wen-An Chiou ${ }^{1}$ \\ 1. AIM Lab, NanoCenter, University of Maryland, College Park, MD 20742-2831, U.S.A
}

Austenitic phase 316L stainless steels (SS) have excellent behavior in corrosion resistance, mechanical strengths and weldability that allows for wide applications. Depends on working temperature, carbides, intermetallic phases, precipitates and martensitic transformation can be present in 316L SS [1]. Although electro-jet-polishing has been a popular technique in preparing SS TEM specimen, selection of proper electrolytes and adjusting etching/polishing conditions can be challenging [2]. Alternatively, SS TEM specimens can also be prepared with ion milling technique, but ion beam heating during $\mathrm{Ar}^{+}$beam bombardment causes artifacts and phase transformation [3]. Thus, it is important to identify a suitable TEM specimen preparation technique for 316L SS. This paper contrasts the application of electro-jetpolishing to ion milling techniques in preparation of 316L SS TEM specimen.

Pieces of 4 mm thick 316L SS (UNS S31603) were cut from a SS cylinder bar (Fig. 1). TEM specimens were initially thinned by mechanical grinding/polishing to about $\sim 100 \mu \mathrm{m}$ in thickness. One specimen was further thinned by a single-jet-electropolisher (SBT model 550) at $15 \mathrm{~V}$ and $-20^{\circ} \mathrm{C}$. The other specimen was ion-milled (Fischione model 1010) with and without liquid nitrogen $\left(\mathrm{LN}_{2}\right)$ cold stage for the purpose of comparing temperature effect on microstructure. TEM analysis was carried out using JEOL $2100 \mathrm{LaB}_{6}$ TEM. The sample was also studied by X-ray diffraction (XRD, Bruker D8 diffractometer).

XRD pattern reveals only pure austenitic phase $(\gamma)$ existed in this 316L SS sample (Fig. 1). Strain contrast (arrows) and parallel plate-like features (open arrows) with 5 $100 \mathrm{~nm}$ in width and few $\mu \mathrm{m}$ in length are clearly shown after electro-jet-polishing (Figs. 2a and 2b). Selected-area diffraction (SAD) pattern confirmed that 316L SS is in austenitic phase. High density of stacking faults (SFs) on $\{111\} \gamma$ plane interior grain (Fig. 2b) and one end pinned at the boundaries (arrows in Fig. 2c) were observed in HRTEM images. The plate-like morphology with a thickness of $2 \sim 3 \mathrm{~nm}$ was identified as twin structures on (111) $\gamma$ plane (Fig. 2c). The large numbers of twin structures observed in HRTEM likely resulted from the high density of stacking faults (SFs) which contained high SF energy (SFE). The thicker twin boundaries (open arrow in Fig. 2c) reflect the higher SFE that remained in the interior of grains. Interestingly, the use of $\mathrm{LN}_{2}$ stage during ion-milling resulted in a $316 \mathrm{~L}$ SS microstructure similar to the electro-jet-polished specimen (with high density SFs and twins with 2 3 nm-thick boundaries). In contrast, specimens prepared by ion-milling without $\mathrm{LN}_{2}$ stage shows decreased SFs density (Fig. 3a) and increased nano-size twin structures (3 8 nm in width) with atomic abrupt boundaries (Fig. 3b). The reduced density of stacking faults and formation of nano-twins caused by ionmilling without $\mathrm{LN}_{2}$ stage can thus be attributed to an effect of low temperature annealing. [4]

\section{References:}

[1] R. A. Covert and A. H. Tuthill, Dairy, Food and Environ. Sanit., 20, 506 (2000).

[2] D.V. Sridhara Rao, K.Muraleedharan and C.J. Humphreys, "TEM specimen preparation techniques", in Microscopy: Sci., Tech., Appl. and Education, ed. A. Méndez-Vilas and J. Díaz, Fromatex (2010).

[3] B. Viguier and A. Mortensen, Ultramircoscopy 87, 123 (2001). 
[4] TEM work was partially supported by NSF-MRSEC (DMR 05-20471) and UMD. 316L SS sample was provided by Dr. Carlos Mario Garzon Ospina.
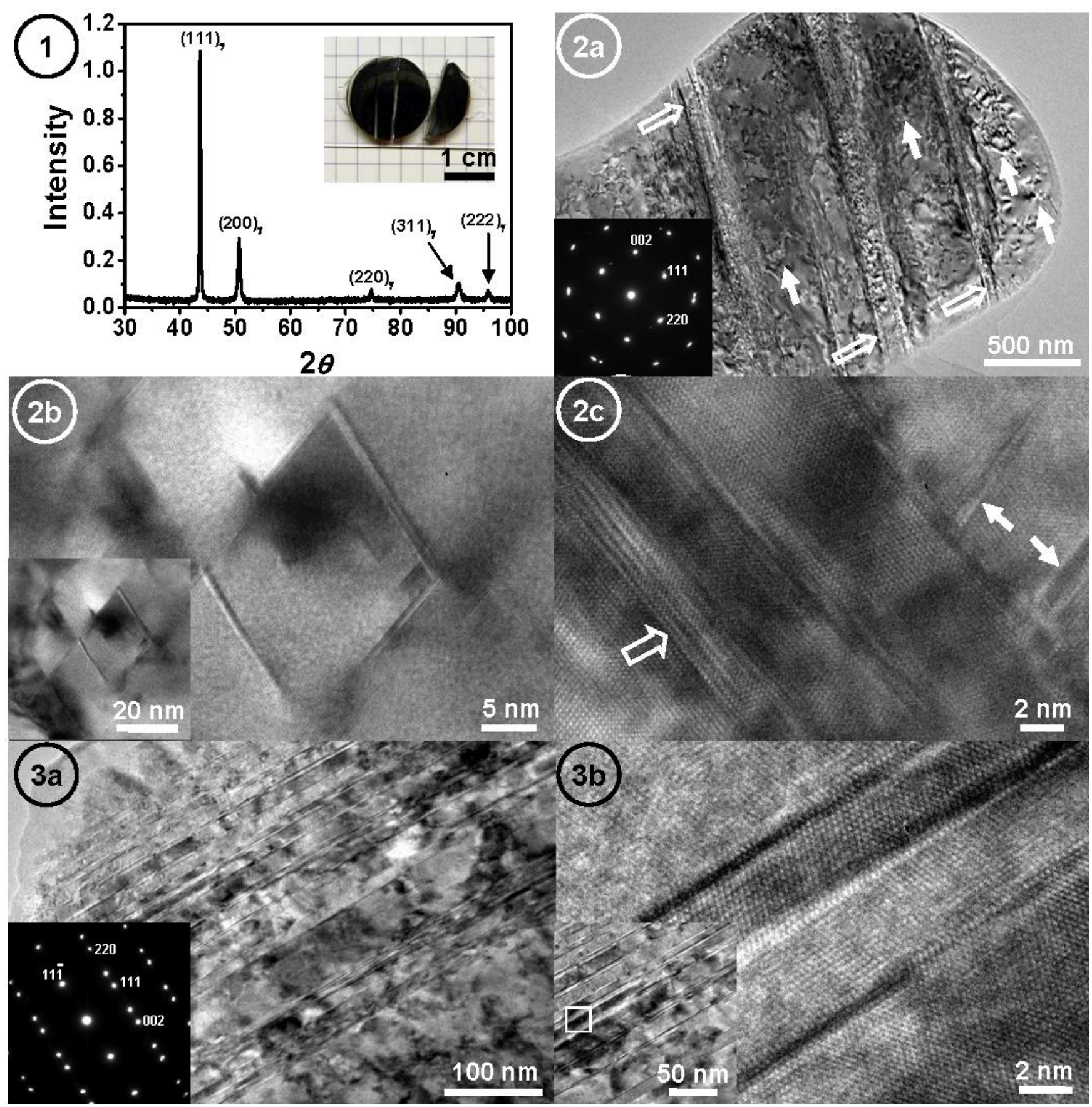

Fig. 1. X-ray diffraction pattern of 316L SS. Inset reveals a photo of 316L SS used in this study.

Fig. 2. TEM images (a, and inset in b), SAD pattern (inset in a) and HRTEM images (b, an enlargement of the inset, and c) of $316 \mathrm{~L} \mathrm{SS}$ prepared by electro-jet-polishing. Strain contrast/SFs areas are shown by arrows. Open arrows point twin boundaries.

Fig. 3. TEM image (a), SAD pattern (inset in a) and HRTEM images (b, an enlargement of the inset) of 316L SS prepared by ion-milling without using $\mathrm{LN}_{2}$ stage. 